\title{
Un contributo allo studio della geografia dei distretti industriali
}

\section{Zusammenfassung}

Die wirtschaftliche Entwicklung der norditalienischen Regionen weist in letzter Zeit einige charakteristische Züge auf, die ein besonderes Territorialmodell darstellen. Es basiert auf einem Industrialisierungsprozeß im peripheren Raum und widerspricht in gewissen Punkten dem klassischen raumwirtschaftlichen Paradigma, das sich vorwiegend auf eine starke Urbanisierung abstützt.

Dieses Produktionsmodell kann kurz folgendermaßen beschrieben werden: Gruppen von kleinen und mittleren Unternehmen, die vorwiegend im ländlichen Raum angesiedelt sind, haben Zonen von integrierter Industrialisierung geschaffen. Die einzelnen Betriebe sind über Zuliefer- und Absatzbeziehungen voneinander abhängig. Auf diese Weise entstehen die sog. "industrial districts". Der Erfolg und die Verbreitung dieser "industrial districts" beruhen auf besonderen geschichtlichen, soziologischen und ökonomischen Voraussetzungen. Sie haben meist an Standorten Fuß gefaßt, wo schon früher das Handwerk neben der landwirtschaftlichen Subsistenzwirtschaft existierte. Es kann nachgewiesen werden, daß sich bereits damals aus Handwerksproduktionsgruppen kleinere Industrien bildeten, für welche die Anfangsinvestitionen nicht allzu hoch waren. Als Fortsetzung dieser Entwicklung finden wir heute diese "industrial districts" fast ausschließlich in den Sektoren der sog. Leichtindustrie (Holz-, Textil- und Lebensmittelindustrie).

Obwohl diese "industrial districts" eine sehr komplexe Innenstruktur besitzen, wurden sie bis heute in Untersuchungen fast immer ausschließlich als einheitliche Räume erfaßt. Aus einer Analyse, basierend auf statistischen Daten und einfachen empirischen Beobachtungen, wird ersichtlich, daß sie ein Phänomen von räumlicher und zeitlicher Dynamik darstellen. Es scheint deshalb zweckmäßig, geographische Untersuchungsmethoden anzuwenden. In diesem Artikel wurde als Untersuchungsraum Ostfriaul gewählt, wo sich die Unternehmen auf etwa 10 Gemeinden mit einer Gesamtfläche von $400 \mathrm{~km}^{2}$ konzentrieren. Es handelt sich dabei um einen mittleren "industrial district" mit etwa 10000 Beschäftigten in 1300 Betrieben, alle fast ausschließlich auf die Herstellung von Holzstühlen spezialisiert, mit einem Umsatz von etwa 1000 Milliarden Lire (Stand 1990). Die Studie hat sich vor allem diese Spezialisierung mit den entsprechenden Zuliefer- und Absatzbeziehungen zur Aufgabe gestellt. Sie kann sicher kein vollständiges Bild vermitteln, erscheinen doch gewisse Produktionsfunktionen teils dispers, teils konzentriert, was mit den unterschiedlichen Standortfaktoren erklärt werden kann. Interessant ist die daraus resultierende Tatsache, daß sich innerhalb dieser «industrial districts" oft "subdistricts" bilden, zwischen welchen sich hierarchische Beziehungsfaktoren etablieren.

\author{
Introduzione
}

Il distretto industriale, altrimenti detto area sistema o distretto marshalliano, caratterizza in modo sempre più rilevante il paesaggio economico e culturale delle paesi industrializzati; per questo motivo esso sta attirando in misura sempre maggiore l'attenzione di studiosi della localizzazione. Il distretto industriale (DI) consiste in una originale struttura territoriale basata sulla diffusione e concentrazione di piccole e medie imprese in un contesto economico-sociale estremamente dinamico.

L'affermazione di questa forma di organizzazione spaziale della produzione quale componente dei sistemi economici ha sconvolto i fondamenti del ragionamento geoeconomico convenzionale: fattori quali la flessibilità dell'organizzazione aziendale, la profonda compenetrazione tra ambiente sociale e comportamento economico, la componente storico-culturale e lo sviluppo tecnologico sfuggono in qualche modo agli schemi della geoeconomia tradizionale. Partendo da queste considerazioni si puó affermare che i DI rappresentano l'aspetto territoriale-economico di una tendenza dell'organizzazione della produzione basata sulla frammentazione e sulla flessibilità aziendale; il nuovo scenario che emerge, qualificato come postfordista, stimola l'elaborazione di una nuova e più adatta metodologia di ricerca geografica.

I DI consistono in un'unità socio-economico-territoriale nell'ambito del quale è difficile scindere le diverse componenti. É per questo motivo che essi rappresentano nell'ambito scientifico un oggetto di studio interdisciplinare, in rapporto al quale tuttavia si può notare come siano stati gli aspetti sociali ed economici ad attirare maggiormente l'interesse scientifico a discapito dell'approccio geografico.

Infatti anche se già MARSHALL alla fine dell'Ottocento aveva descritto le esternalities che caratterizzano la localizzazione industriale, i DI modernamente intesi sono stati individuati e teorizzati da sociologi ed economisti: in particolare il sociologo BAGNASCO ha rilevato nella diffusione delle piccole e medie imprese una delle principali componenti della cosiddetta III Italia (BAGNASCO 1977); successivamente gli economisti hanno evidenziato gli aspetti produttivi ed organizzativi dei DI partendo da

Igor Jelen, Dipartimento di Scienze Politiche, Università degli Studi di Trieste, Piazzale Europa 1, 34100 Trieste. 
questi presupposti (BECATTINI 1987, FUA e ZACCHIA 1983). Al di la di questi approcci che sostanzialmente prescindono dalla componente territoriale, alcuni autori hanno recentemente dimostrato che il metodo geografico può essere utilmente impiegato per l'analisi del DI (SCOTT 1992, SCOTT-KWORK 1989); si può dire che in questo senso si stanno orientando gli studi recenti sulla localizzazione industriale.

Il DI sta assumendo quindi un ruolo innovativo nel contesto paradigmatico geoeconomico e per questo motivo esso si pone in modo alternativo rispetto ai tradizionali modelli sospesi tra la politica dei grandi investimenti in poles de croissance di PERROUX e politiche liberiste (SCOTT 1986, LODA 1989, HARRISON 1992). Il ragionamento economico convenzionale ne risulta mutato: esso si basava sull'assunto dell'homo oeconomicus che razionalizza il proprio comportamento fino al punto da costruirsi uno schema di comportamento distinto per ciascuna delle proprie attività. Cio ha portato all'elaborazione di una metodologia basata su atomizzazione e parcellizzazione dei comportamenti sociali ed economici. Si tratta di assunzioni necessarie in quanto solamente grazie ad esse è possibile, in base al ragionamento classico, rendere la realtà conoscibile: isolare le variabili è un presupposto per poter applicare il principio della causalità in tutta la sua potenza.

Ma questo metodo si è dimostrato illusorio in geografia cosi come nelle altre scienze umane: il comportamento economico non puó essere distinto dal contesto geografico-sociale (BÖSCH 1987). La comprensione del fenomeno DI non può avvenire facendo ricorso alla teoria geoeconomica classica in quanto consiste in una realta complessa dove le diverse componenti socio-economiche risultano essere intrinsecamente connesse. Quale approccio metodologico appropriato per il DI è stato recentemente proposto un metodo che si basa sullo studio contestuale delle diverse componenti socio-economiche quale l'embedding di GRANOVETTER (riproposto da HARRISON 1992).

Secondo la definizione comune il DI consiste in un insieme di aziende dalle dimensioni medie e piccole localizzate in una data area la cui attività afferisce al medesimo ciclo di produzione. Cosi teorizzato il DI rappresenta senz'altro un'astrazione: nella realtà esso appare sul territorio come un sistema misto dove diversi cicli di produzione si sovrappongono e creano situazioni complesse. Inoltre le piccole e medie aziende sono per loro stessa natura instabili e precarie in termini di gestione, di mercato e di sviluppo (TRUFFELLI 1989); resta comunque necessario l'assunto dell'unicita del ciclo di produzione quale riferimento simulativo della ricerca.

Il DI rappresenta un fenomeno territoriale per lo studio del quale si può disporre di una metodologia consolidata; in termini geografici esso rappresenta una regione in quanto consiste in un'area in cui una data variabile (ad es. la distribuzione di aziende afferenti allo stesso ciclo di produzione) è distribuita in modo omogeneo e prevalente. Può essere oggetto quindi di tutta una serie di categorie concettuali elaborate dalla moderna analisi localizza- tiva in quanto si presume che esistano all'interno della regione geografica DI strutture territoriali quali la struttura centro-periferia, processi di diffusione, gerarchie, flussi ed interdipendenze spaziali e cosi via.

La formazione di un DI può essere definita come un processo fondamentalmente spontaneo almeno per quanto riguarda i distretti storicamente formatisi nella III Italia di BAGNASCO. Questi infatti risalgono ad una situazione economica e sociale premoderna, in cui l'attività artigianale svolgeva il ruolo di attività complementare ad una attività agricola di pura sussistenza. Successivamente, in particolari situazioni storiche, l'attività artigianale si è affermata come industria leggera che si è quindi adeguata alla nuova organizzazione economica basata sulla produzione di beni di consumo.

Diversi sono i prerequisiti ambientali che vengono di volta in volta identificati come essenziali per la formazione di un DI. Secondo alcuni autori questi sono potuti emergere in situazioni sociali caratterizzate da comunità rurale e famiglia allargata in cui forte era sia lo spirito della solidarieta sociale che l'effetto di emulazione ed imitazione. Altri hanno sottolineato l'effetto delle economie di scala della produzione in seguito a mutamenti strutturali che hanno portato ad un nuovo modello produttivo definito come postfordista; ancora si è argomentato circa elementi di carattere culturale ed antropologico che caratterizzano i modelli comportamentali degli imprenditori. $\dot{E}$ essenziale tuttavia sottolineare che alla base della formazione di ogni DI vi è una storia di fatti sociali, economici e culturali geograficamente localizzati. Si tratta ora di capire in che misura i singoli DI possano essere interpretati come casi particolari (sulla base dell'exeptionalism di SCHÄFER) ovvero come eventi teorizzabili e quindi riproducibili. In effetti i DI rappresentano fondamentalmente delle eccezioni nella storia economica e territoriale: nella maggior parte dei casi aree di specializzazione e concentrazione artigianale non si sono evolute in moderni DI. Basti citare nel Friuli-Venezia Giulia (Italia nord-orientale) i cestai di Pieris d'Isonzo, i mobilieri di Sutrio, i pantofolai di S. Daniele o le coltellerie di Maniago: in questi casi un solido potenziale di conoscenze artigianali non si è tramutato o si è tramutato solo in parte in un DI. Al contrario questa trasformazione è avvenuta con successo nella stessa regione per l'area della produzione della sedia nel Manzanese. É probabile che non esista una spiegazione perfettamente monodisciplinare a questo fatto; la teorizzazione geografica consente tuttavia un approccio forse piu completo in quanto assume la dimensione territoriale come sintesi di tutte le componenti rilevanti nella formazione dei fenomeni.

\section{Proposito}

Nonostante l'importanza che il DI sta assumendo nel quadro delle moderne teorie di localizzazione, la struttura territoriale degli stessi rimane qualcosa di fondamentalmente sconosciuto: il DI è stato infatti assunta come un quantum territoriale piuttosto che come fenomeno 
caratterizzato da struttura e dinamica interne. Le eccezioni hanno tracciato la via da seguire: esse si basano sullo studio della correlazione tra distanza (qualsiasi sia il significato geografico che a questo concetto vogliamo dare) e fenomeni economici quali possono essere ad es. le dinamiche della disintegrazione produttiva, la formazione di nuove aziende, i flussi di servizi e beni, il rapporto fornitori-clienti (SCOTT-KWORK 1989, PYKE-BECATTINISENGENBERGER 1991).

Questo contributo si propone di indagare i meccanismi intrinseci di questa struttura alla ricerca di regolarità spaziali: assunto il DI come un modello astratto si tratta quindi di verificarne le caratteristiche strutturali e di processo. Non è quindi intenzione considerare l'ambiente globale in cui i DI si sviluppano, ad es. temi di studio quali la riconversione del paradigma dello sviluppo o altre macrotendenze. E invece interesse di questo studio conoscere in maniera piu particolareggiata le dinamiche interne con lo scopo di verificare la possibilita di riprodurre il modello in altre situazioni geografiche e, in ultima analisi, creare le condizioni perchè esso possa divenire uno strumento di politica territoriale.

La regione geografica definita come DI rappresenta un microcosmo dove la localizzazione e lo sviluppo aziendale avvengono in base a criteri particolari. L'elemento fondamentale che qualifica un DI è il ciclo di produzione caratterizzato da una sequenza di fasi di lavorazione. Queste fasi possono essere localizzate presso una azienda in modo singolo o a gruppi: esistono quindi aziende integrate, dove il ciclo di produzione viene svolto in modo più o meno completo, oppure aziende specializzate. Il ciclo di produzione è suscettibile di disintegrazione ovvero integrazione; questo processo genera continuamente nuove aziende o nuove unita produttive in un ambiente estremamente dinamico. La sequenza di funzioni in cui puo essere scomposto il ciclo di produzione può essere definito come un flusso di beni e servizi caratterizzato da un verso e da un'intensità. É quindi fondamentale capire se la distribuzione sul territorio delle funzioni avviene in modo uniforme ovvero se esistono delle aree di accumulazione. In quest'ultimo caso si potrebbe verificare la presenza di una struttura gerarchica sulla base della quale in diverse aree, ovvero in diverse sottoaree appartenenti allo stesso DI, vengono localizzate fasi diverse dello stesso ciclo di produzione.

\section{Caso di studio: L'area della sedia nel Friuli orientale}

Il seguente studio si inserisce in un più ampio progetto di ricerca che si propone la ricostruzione tramite mezzi di geografico-informativi dello scenario del settore dell'industria del legno nella regione Friuli-Venezia Giulia con l'obiettivo di individuarne le tendenze di sviluppo territoriale.

L'area di studio consiste nel DI della cosiddetta «area della sedia" localizzato nel Friuli orientale in un'area essenzialmente rurale situata grosso modo a metà strada tra i
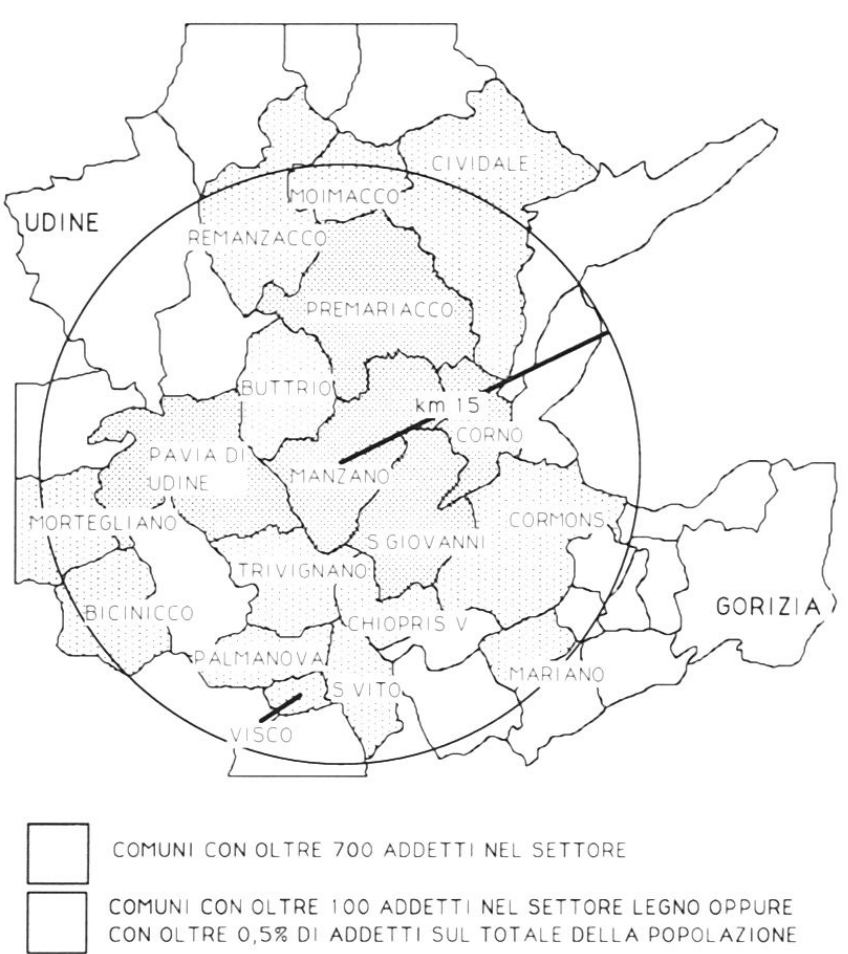

Figura A Delimitazione del distretto industriale della sedia nel Friuli orientale.

capoluoghi provinciali di Udine e Gorizia. L'area della sedia costituisce un caso classico di DI: le attività connesse in qualche modo al ciclo di produzione di sedie in legno costituiscono l'attività economica predominante. La concentrazione di piccole e medie imprese ha raggiunto livelli estremi, basti pensare che circa il 50\% della produzione di sedie in legno nella Comunità europea, corrispondente a circa l' $80 \%$ di quella italiana, ha luogo sul territorio dei 7-8 piccoli comuni che vengono generalmente identificati con l'area della sedia.

In particolare il DI del Friuli orientale puo essere assimilato ad una regione geografica composta di comuni di Manzano, S. Giovanni al Natisone, Corno di Rosazzo, dove l'attivita si è originariamente diffusa, e quindi dai comuni di Premariacco, Pavia di Udine e altri comuni minori limitrofi agli stessi; esso occupa una superficie di ca. $400 \mathrm{~km}^{2}$ con una popolazione che non supera i 40000 abitanti. La struttura industriale-artigianale classificata come ramo ISTAT 46 "Industrie del legno e del mobile in legno" è costituita da circa 1300 aziende per un totale di ca. 10000 addetti (il 25\% dell'intera popolazione) (tabella 1).

L'attuale DI della sedia è il risultato di un complesso processo di sviluppo regionale che offre diverse prospettive di studio: da quella storica (EXNER 1879, BOSCO-DEGANUTTI 1986) a quella sociale (MATTIONI-BEDNARZ 1985) e

Istituto centrale di statistica, Roma 
Fonte: a) elaborazione dati ISTAT; b) banca dati CERVED

Tabella 1 Comuni del distretto industriale della sedia. Numero addetto per il ramo ISTAT 46.

a quella geo-economica. Diversi inoltre sono le pubblicazioni che riportano dati e informazioni strutturali sul DI; si tratta in genere di enti pubblici, quali le associazioni di categoria o la Camera di Commercio, che sono istituzionalmente deputati alla raccolta di informazioni economiche ma che non hanno particolare interesse per aspetti teoretici (ESA 1982). Si può affermare quindi che l'analisi della struttura geografica del DI resti un argomento di ricerca in parte inesplorato.

\section{Il metodo}

Il DI del Friuli orientale risulta essere una regione geografica dai limiti ben definiti entro i quali la localizzazione delle aziende assume una forma grosso modo concentrica. In pratica l'insieme delle circa 1300 aziende può essere assimilato ad un'unica grande unità produttiva dove le diverse funzioni di produzione vengono disperse sul territorio in base ad un processo di disintegrazione.

Il primo problema che si pone risulta quindi essere quello di creare una tassonomia delle aziende e delle relative funzioni con lo scopo di realizzare l'equazione localizzazione $=$ funzione aziendale sul territorio esaminato; suc- cessivamente sarà possibile ricostruire ed analizzare la struttura geografica della produzione su scala regionale.

La classificazione risulterà necessariamente approssimata: qualsiasi ciclo di produzione infatti puo essere scomposto in un numero indefinito di fasi a seconda dell'evoluzione tecnologica o della situazione congiunturale delle curve di costo che sono alla base dei processi disintegrativi; inoltre la distinzione tra diverse fasi del ciclo è difficile per certi tipi di prodotto oltre che di lavorazione. Tutto ciò è comprensibile considerando che l'ambiente delle piccole e medie imprese è strutturalmente precario ed è caratterizzato da fenomeni reversibili di disgregazione ovvero integrazione produttiva.

$\mathrm{Nel}$ caso in parola si è proceduto alla classificazione sequenziale che appare nelle tabelle 2 e 3 . In particolare la categoria PRODUZIONE comprende le aziende che trattano e commercializzano il prodotto finito; non si tratta sempre di aziende fortemente integrate ma in ogni caso di aziende che coordinano e gestiscono l'intero ciclo di produzione. Spesso tali aziende dispongono di un catalogo dei prodotti con relativi prezzi al pubblico. Le categorie ELEMENTI (semilavorati) e LAVORAZIONI riguardano le aziende terziste che non è stato possibile definire altrimenti e che sono orientate rispettivamente alla produzione di elementi e a lavorazioni generiche. Generalmente questi imprenditori non dispongono di una gamma definita di prodotti e prezzi, ma concordano il prezzo sulla base di uno schema di preventivo. Il significato delle altre categorie riportate dalle tabelle 2 e 3 è evidente; esse riguardano lavorazioni ben definite nell'ambito della tecnologia del legno.

La classificazione delle aziende (tabella 3 ) è avvenuta sulla base della descrizione della ragione sociale ottenuta dal Registro Ditte fornito dalla Camera di Commercio. Si tratta di descrizioni spesso ambigue in quanto rappresentano piu che altro delle dichiarazioni di intenti da parte degli imprenditori e degli artigiani oppure delle manifestazioni di ciò che essi ritengono di essere. Per apportare dei miglioramenti alla classificazione sono state visitate 100 aziende (il $8 \%$ del totale, per i dettagli di questa ricerca si veda JELEN 1991) nell'autunno 1991 per le quali si è tentato di determinare nel modo più esatto possibile l'articolazione del ciclo di produzione all'interno dell'azienda. Il metodo di classificazione è stato verificato quindi sulla base di questo campione. Altri casi dubbi sono stati chiariti tramite telefonate oppure sono stati tralasciati e

1) ACQUISTO MATERIE PRIME

2) SEGHERIA

3) ELEMENTI (SEMILAVORATI)

4) PRODUZIONE $\Rightarrow$ LAVORAZIONI VARIE (CARTEGGIATURA,

5) COMMERCIALIZZAZIONE $=>$ MERCATO 


\begin{tabular}{|c|c|c|c|c|c|c|c|c|}
\hline & $\begin{array}{l}\text { CORNO DI } \\
\text { ROSAZZO }\end{array}$ & MANZANO & $\begin{array}{l}\text { PAVIA DI } \\
\text { UDINE }\end{array}$ & PREMARIACOO & $\begin{array}{l}\text { S.GIOVANNI } \\
\text { AL NATISONE }\end{array}$ & TOTALE & CHI QUADFATO & SIGNIFICATIVITA' \\
\hline 1 вемеNTI & 34 & 80 & 17 & 19 & 82 & 232 & 8,972 & 0.062 \\
\hline 2 PROOUZONE & 25 & 49 & 2 & 18 & 40 & 134 & 17,464 & 0.002 \\
\hline 3 CARTEGGIATURA & 2 & 7 & 2 & 1 & 5 & 17 & & \\
\hline 4 LAVORAZIONE & 3 & 4 & 10 & 18 & 32 & 67 & 32,088 & 0.000 \\
\hline 5 FALEGNAMERIA & 4 & 1 & 5 & 17 & 6 & 33 & & \\
\hline 6 INFANZIA & 2 & 4 & 4 & 0 & 5 & 15 & & \\
\hline 7 LEVIGATURA & 10 & 43 & 7 & 11 & 41 & 112 & 3,439 & 0.487 \\
\hline 8 IMPAGLIATUPA & 3 & 8 & 4 & 4 & 6 & 25 & & \\
\hline 9 ASSEMBLAGGIO & 4 & 14 & 4 & 1 & 4 & 27 & & \\
\hline 10 TORNITUPA & 3 & 10 & 2 & 4 & 18 & 37 & & \\
\hline 11 VEPNICIATURA & 4 & 35 & 5 & 18 & 53 & 115 & 12,402 & 0.015 \\
\hline 12 SEGHERAA & 7 & 26 & 13 & 9 & 21 & 76 & 8,298 & 0.081 \\
\hline 13 COMPENSATO & 0 & 3 & 1 & 0 & 2 & 6 & & \\
\hline 14 INCANNETTATURA & 0 & 5 & 0 & 7 & 6 & 18 & & \\
\hline 15 CIPPATURA & 0 & 1 & 0 & 0 & 2 & 3 & & \\
\hline 16 COMMEACIALE & 0 & 5 & 3 & 0 & 1 & 9 & & \\
\hline 17 CURVATI & 0 & 3 & 0 & 2 & 5 & 10 & & \\
\hline 18 ESICCAZIONE & 0 & 0 & 0 & 0 & 3 & 3 & & \\
\hline 19 LAVOR GIUNCO & 0 & 0 & 2 & 0 & 0 & 2 & & \\
\hline 20 IMBALAGGIO & 0 & 2 & 0 & 2 & 0 & 4 & & \\
\hline 21 PANTOGRAFATURA & 0 & 5 & 1 & 2 & 4 & 12 & & \\
\hline 22 ALTAO & 2 & 7 & 2 & 1 & 2 & 14 & & \\
\hline TOTALE & 103 & 312 & 84 & 134 & 338 & 971 & & \\
\hline $1+9+4$ & 41 & 98 & 31 & 38 & 118 & 326 & 2,986 & 0.560 \\
\hline $3+7$ & 12 & 50 & 9 & 12 & 46 & 129 & 3,908 & 0.419 \\
\hline $4+5$ & 7 & 5 & 15 & 35 & 38 & 100 & 63,944 & 0.000 \\
\hline $14+8$ & 3 & 13 & 4 & 11 & 12 & 43 & 5,926 & 0.205 \\
\hline
\end{tabular}

Fonte: banca dati CERVED 


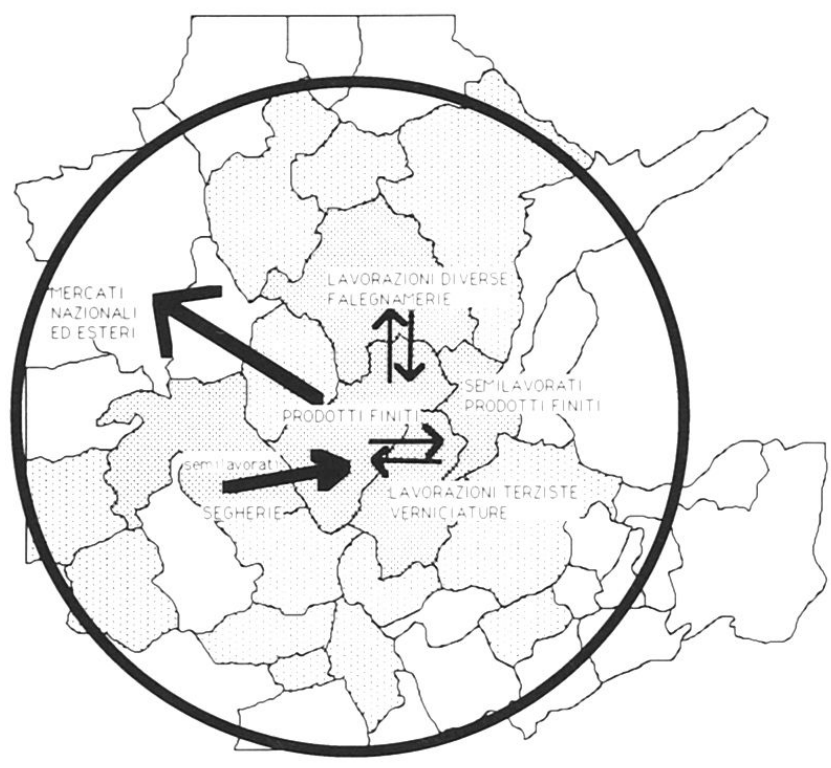

Figura B Sottoaree di specializzazione all'interno del distretto industriale della sedia.

classificati come $A L T R O$. É necessario notare come per esigenze di semplificazione della ricerca ciascuna azienda è stata ricondotta ad un'unica funzione, seppure per ridurre l'imprecisione sono state assunte categorie ampie (quali ad es. le categorie SEMILAVORATI, PRODUZIONE o LAVORAZIONI); d'ora in avanti si parlerà quindi di aziende-funzione. Per altri motivi non è stato possibile ricorrere alla classificazione ISTAT, e cioè del ramo no 46 ; infatti tali classi non rispondono all'esigenza di classificare in modo sequenziale il ciclo di produzione per poter determinare l'equazione luogo = funzione produttiva come sopra presupposto.

La classificazione territoriale è stata effettuata sulla base dei 5 comuni di Manzano, S. Giovanni al N., Pavia di U., Corno di R. e Premariacco nei quali si concentra oltre il $70 \%$ del numero complessivo delle aziende operanti nel DI. La ripartizione amministrativa è fonte di bias in quanto è dubbio che i comuni rappresentino delle classi naturali per la distribuzione delle aziende-funzione; la diffusione all'interno dell'area non sembra infatti essere molto sensibile all'effetto barriera esercitato dai confini comunali. Tale ripartizione e tuttavia necessaria in quanto i dati ISTAT sono organizzati su base comunale.

Allo stesso modo è senza dubbio fonte di bias l'assunzione delle aziende come variabile descrittiva invece della variabile addetti; la ragione di questa opzione sta nell'assumere l'azienda come elemento chiave dello sviluppo territoriale al di là del significato qualitativo-dimensionale che caratterizza il singolo caso. In altre parole la formazione di un'azienda è indice di sviluppo strutturale sul territorio in quanto alla base della stessa vi è un patrimonio di conoscenze ed esperienze e di tutto cio che è connesso con il concetto dell'imprenditorialità. La dimensione in termini di addetti invece puó riflettere situazioni congiunturali.

\section{Analisi}

Primo obiettivo della ricerca consiste ora nell'accertare in che misura la distribuzione di aziende-funzioni sia omogenea sul territorio ovvero, in altre parole, si vi è concentrazione o dispersione delle aziende-funzione tra $\mathrm{i}$ comuni selezionati. Entrambe le possibilità implicano conseguenze geo-teoretiche interessanti.

Nel primo caso si assume che non vi sia autocorrelazione spaziale tra le localizzazioni delle aziende e quindi non vi siano concentrazioni e fenomeni di specializzazione spaziale. La distribuzione regolare sul territorio significa anche che le diverse funzioni si localizzano in modo tale da minimizzare la distanza tra le aziende di categoria diversa; le funzioni sono quindi spazialmente interdipendenti.

Nel secondo caso invece si assiste alla formazione di cluster funzionali: gruppi di aziende-funzione vengono localizzate prevalentemente in determinate aree ed esisterebbe quindi specializzazione territoriale. In questo caso la concentrazione-specializzazione spaziale sarebbe indice di fattori di localizzazione particolari all'interno del DI la cui individuazione rappresenta un obiettivo molto interessante della ricerca.

Nella realtà è improbabile che venga individuato uno di questi due modelli nella forma pura: le diverse funzioni sono caratterizzate da modalita localizzative peculiari a quel tipo di tecnologia ed organizzazione aziendale. Ad es. per quanto riguarda l'area della sedia, la localizzazione delle attivita imprenditoriali può dipendere da diversi fattori quali le barriere all'entrata, necessita di effettuare investimenti rilevanti, e quindi costi fissi e alto standard tecnologico. Ancora, è presumibile che la localizzazione di attività imprenditoriali di organizzazione relativamente semplice, come ad es. la levigatura, la carteggiatura, l'assemblaggio, sia piu sensibile alla localizzazione dell'impresa committente, questo per minimizzare la distanza dalle stesse. Al contrario per attivita più particolari che richiedono manodopera specializzata o macchinari costosi, ad es. i compensati multistrati, è probabile che economie di localizzazione si sviluppino in determinate aree.

L'analisi dei dati grezzi lascia immaginare che per le diverse funzioni vi siano delle concentrazioni nei 5 comuni considerati. É necessario tuttavia ricorrere ad uno strumento di analisi più raffinato in quanto le numerosità per funzione e comuni non sono sempre significative; inoltre è difficile valutare i diversi valori in riferimento al peso relativo della funzione e del comune. Un test deve essere quindi effettuato per verificare l'esistenza o meno di omogeneità all'interno del DI a prescindere dal peso delle singole classi. In altre parole si tratta di verificare se i comuni si specializzano per quanto riguarda le diverse funzioni oppure no prescindendo da ciò che appare dall'analisi dei dati grezzi.

Per svolgere questa verifica si procede all'analisi della tabella delle frequenze ed al computo del test non parametrico del chi quadrato. Questo costituisce uno strumento sensibile per individuare differenziazione a prescindere 
dalle grandezze assolute delle categorie: la quantità, in questo caso il numero di aziende-funzioni per comune, viene relativizzata con riferimento al valore atteso di aziende-funzioni ricavato in base alla dimensione assoluta della funzione nell'intero DI (per semplicità assimilato ai 5 comuni più rappresentativi). Nel caso dell'area della sedia il parametro di ponderazione assunto risulta essere di 321 per Manzano, 348 per S. Giovanni al Nr., 134 per Premariacco, 106 per Pavia di U. e 84 Corno di R. Questi valori corrispondono al rapporto tra i totali di aziende operanti nel settore del legno (ramo ISTAT no 46) per i 5 comuni considerati come ricavato dalla tabella 3 . In pratica esso consiste in un rapporto di ponderazione che permette di definire il valore atteso e confrontarlo con il valore osservato della presenza delle aziende-funzioni.

Qualora non vi fosse differenza significativa tra valore atteso e valore osservato, si potrebbe concludere che il DI è strutturalmente omogeneo (ipotesi nulla). Al contrario, qualora la differenza fosse significativa, si potrebbe concludere circa la presenza di aree di accumulazione di aziende-funzioni. Le classi assunte sono state in alcuni casi compattate con il fine di aumentare il numero di casi per poter rendere significativo il test. Questa operazione è stata possibile solamente per classi di funzioni analoghe sia come metodo di lavorazione sia come modalita di gestione come ad es. IMPAGLIATURA, CARTEGGIATURA e LEVIGATURA.

Come si puo notare una varianza apparentemente notevole per alcune funzioni, non risulta essere significativa sulla base del test. In particolare le aziende classificate come ELEMENTI, PRODUZIONE, LAVORAZIONE, VERNICIATURA dimostrano di essere concentrate piuttosto che disperse (significatività molto bassa). Lo stesso puó essere detto per quanto riguarda le aziende-funzione TORNITURA e FALEGNAMERIA; per queste ultime tuttavia non è possibile procedere al test del chi quadrato a causa dell'esiguo numero di casi. La concentrazione in questi casi rappresenta tuttavia un caso evidente se vengono considerate le cifre assolute ma non è opportuno interpretare in senso inferenziale questi dati: in altre parole la concentrazione di queste aziende-funzione sul territorio potrebbe rappresentare solamente un fatto eccezionale o effimero.

La concentrazione di aziende-funzione appare in alcuni casi particolarmente interessante: per le categorie $P R O$ DUZIONE, ELEMENTI e SEGHERIA si puo configurare addirittura la formazione di sottoaree sistema, tale è la predominanza di questi tipi di aziende in alcuni comuni. In particolare la prima categoria che rappresenta soprattutto aziende orientate alla produzione ad alla commercializzazione del prodotto finito sembra essere localizzate in modo predominante nel comune di Manzano. Si tratta di aziende con organizzazione e dimensione aziendale più complesse, con listini prezzi e una rete di rapporti commerciali (categorie 1 e 2 secondo la definizione JELEN 1991).

La categoria ELEMENTI è predominante invece nel comune di S. Giovanni al N. Si tratta della categoria alquan- to eterogenea di aziende terziste il cui sviluppo è dovuto soprattutto al fenomeno della disintegrazione produttiva che avviene nel contesto delle aree sistema. La presenza e predominanza di questo tipo di aziende lascia apparire l'intera struttura produttiva del comune come in qualche modo indotta dalla presenza delle aziende di tipo PRODUZIONE esistenti nel contiguo comune di Manzano. Al contrario le aziende-funzione LEVIGATURA e le combinazioni $14+8$ e $3+7$ (tabella 3 ) risultano essere distribuite omogeneamente nei 5 comuni (significatività del test alta). Si tratta di lavorazioni elementari che richiedono minimi investimenti e bassa specializzazione della manodopera. La formazione delle stesse aziende avviene generalmente come risultato della disintegrazione produttiva: aziende di dimensione media espellono lavorazioni con il fine di minimizzare la dimensione e quindi ridurre i costi fissi. Il fattore determinante della localizzazione di queste aziende è quindi la contiguità alle aziende committenti. Ne deriva una distribuzione territoriale abbastanza uniforme; si tratta in altre parole di aziende autenticamente subordinate nei confronti delle aziende più grandi e complesse.

Per le altre categorie di aziende-funzione non è possibile concludere alcunché considerata l'esiguità dei casi. Peraltro variazioni repentine e rilevanti di queste categorie potrebbe prefigurare nuove forme di accumulazione territoriale.

\section{Conclusioni}

La struttura del DI risulta essere complessa: vi sono aree di specializzazione anche se diverse categorie di aziendefunzione sembrano essere localizzate indifferentemente sul territorio. Emergono addirittura forme territoriali che possono essere definite come "sottodistretti industriali»; questi sono indici della presenza di economie di localizzazione in quanto consistono in zone di accumulazione di funzioni. Su questa base è possibile affermare che i diversi comuni assumano una struttura differenziata: Pavia di U. viene identificata come il centro delle segherie, S. Giovanni al N. come centro delle aziende terziste e delle lavorazioni generiche, Manzano come l'autentico centro delle aziende produttive e commerciali. I comuni di Corno di R. e Premariacco presentano invece una struttura non ben definita.

La specializzazione territoriale che si puó notare corrisponde a fasi diverse del ciclo di produzione; assumendo lo stesso come un flusso di beni e servizi orientato sul territorio ne deriva l'ipotesi che la specializzazione territoriale implichi o venga implicata da una gerarchia interna al DI. Nel caso dell'area della sedia nel Friuli orientale tale gerarchia si sviluppa sull'asse che ha agli estremi le segherie di Pavia di U. e le aziende integrate di Manzano passando per i terzisti di S. Giovanni al N.

Il DI si configura quindi come un fenomeno geografico articolato di cui è interessante analizzare le dinamiche e 
le componenti intrinseche per poter comprenderne le tendenze evolutive nel tempo e nello spazio. In questo modo sarà possibile valorizzare la piccola e media impresa in senso geografico. In questo senso e cioè come riferimento-contesto territoriale di nuove iniziative economiche, il DI potrà essere adattato come strumento di pianificazione territoriale.

\section{Note bibliografiche}

BAGNASCO, A. (1977): Tre Italie. La problematica territoriale dello sviluppo italiano. Bologna.

BAGNASCO, A., TRIGILIA, C. (a cura di) (1984): Società e politica nelle aree di piccola impresa. Il caso di Bassano. Fondazione Corazzin. Arsenale Editrice. Ires Veneto, Venezia.

BAZO, G. (a cura di) (1978): Ricerche socio-economiche per la provincia di Pordenone (4 volumi), CCIAA, Pordenone.

BOESCH,M. (1989): Engagierte Geographie. Zur Rekonstruktion der Raumwissenschaft als politikorientierte Geographie. Franz Steiner Verlag, Stoccarda.

BONETTI, E. (1961): La teoria della localizzazione. Università di Trieste.

CAMERA DI COMMERCIO INDUSTRIA ARTIGIANATO AGRICOLTURA DI UDINE (1989): Situazione economica della provincia di Udine.

EXNER, G. F. (1879): Il villaggio di Mariano nel Friuli austriaco. Gradisca.

FUÀ, G., ZACCHIA, C. (a cura di) (1983): Industrializzazione senza fratture. II Mulino, Bologna.

GRANDINETTI, R. (ricerca coordinata da) (1989): Artigianato e politiche regionali in Friuli Venezia Giulia. Ecipa Friuli Venezia Giulia.

HARRISON, B. (1992): Industrial Districts: Old Wine in New Bottles? In: Regional Studies, no 5, pp. 469-483.

JELEN, I. (1991): L'area della sedia nel Friuli orientale. L'areasistema come paradigma di organizzazione territoriale della produzione. Università di Trieste.

LODA, M. (1989): Das "Dritte Italien". Zu den Spezifika der peripheren Entwicklung in Italien. In: Geographische Zeitschrift, no 3, pp.180-194.

LÖSCH, A. (1940): Die räumliche Ordnung der Wirtschaft. Fischer, Jena.
MATTIONI, F., BEDNARZ, F. (1985): Seggiolai e mercati. Postindustriale e servizi reali in una tipica area-sistema: il Manzanese. Cooperativa Editoriale "ll Campo", Udine.

MICELLI, F. (1991): Identità dei friulani e le sfide del futuro. In: Julia Augusta, no 1, pp. 21-29.

PAGNINI, P. (1989): Riflessioni geografiche per lo studio del territorio. Università di Trieste.

PROMOSEDIA, BANCA POPOLARE DI CIVIDALE (1988): L'universo Promosedia CCIAA, Udine.

PYKE, F., BECATTINI, G., SENGENBERGER, W. (1991): Distretti industriali e cooperazione fra imprese in Italia. Studi e informazioni, Quaderni 34, Supplemento no 1 al no 3. Banca Toscana, Firenze.

REGIONA AUTONOMA FRIULI-VENEZIA GIULIA, ESA - ENTE PER LO SVILUPPO DELL'ARTIGIANATO DEL FRIULI-VENEZIA GIULIA (1982): Indagini sulle aziende produttrici di sedie e affini nel Friuli orientale. Trieste.

REGIONA AUTONOMA FRIULI-VENEZIA GIULIA, IRES FRIULI-VENEZIA GIULIA (1989): II terziario industriale nel Friuli-Venezia Giulia. Trieste.

RIBI, CARLO, A. (composto ed edito da) (1885): Ragguaglio annuale della i. r. scuola d'industria legnaiuola, con annesso corso serale di perfezionamento in Mariano per l'anno scolastico 1884-1885, Gorizia.

RULLANI, E. (1990): Conoscenza e identità: problemi strategici dello sviluppo industriale in Friuli. In: Impresa e sviluppo no 4-5, pp. 20-35

SCOTT, A., STORPER, M., editors (1986): Production, Work, Territory. George Allen and Unwin, Boston.

SCOTT, A. J. (1986): Industrial Organisation and Location: Division of Labor, the Firm, and Spatial Process. In: Economic Geography, no 3, pp. 215-231.

SCOTT, A. J., KWORK, E. C. (1989): Inter-Firm Subcontracting and Locational Agglomeration: A Case Study of the Printed Circuits Industry in Southern California. In: Regional Studies, no 5, pp. 405-416

SCOTT, A. J., ALLEN, J. (1992): The Role of Large Producers in Industrial Districts: A Case Study of High Technology Systems Houses in Southern California. In: Regional Studies, no 3, pp. 265-275.

STORPER, M. (1992): The Limits to Globalization: Technologies Districts and International Trade. In: Economic Geography, no 1, pp. 60-93.

TRUFFELLI, C. (1989): Innovazione, imprese e "ambiente" in tre aree metropolitane del Nordest-Centro. In:Bollettino della Società Geografica Italiana, pp. 227-265.

VALUSSI, G. (1963): Note geografiche sull'artigianato friulano: i seggiolai di Manzano e i coltellinai di Maniago. In: Rivista Geografica Italiana, LXX, pp. 26-59. 